\title{
Selective Degradation of Mitochondria by Mitophagy in Pathogenic Fungi
}

\author{
Jane Sadhna Jagernath, Shuai Meng, Jiehua Qiu, Huanbin Shi, Yanjun Kou* \\ State Key Laboratory of Rice Biology, China National Rice Research Institute, Hangzhou, China \\ Email: *kouyanjun@caas.cn
}

How to cite this paper: Jagernath, J.S., Meng, S., Qiu, J.H., Shi, H.B. and Kou, Y.J. (2021) Selective Degradation of Mitochondria by Mitophagy in Pathogenic Fungi. American Journal of Molecular Biology, 11, 15-27.

https://doi.org/10.4236/ajmb.2021.111002

Received: November 3, 2020

Accepted: December 28, 2020

Published: December 31, 2020

Copyright $\odot 2021$ by author(s) and Scientific Research Publishing Inc. This work is licensed under the Creative Commons Attribution International License (CC BY 4.0).

http://creativecommons.org/licenses/by/4.0/

\begin{abstract}
Selective mitochondrial autophagy or mitophagy is an evolutionarily conserved cellular process that selectively degrades superfluous, damaged, and dysfunctional mitochondria. This process is believed to be a mitochondrial quality control system crucial for intracellular homeostasis. Recently, researchers developed a range of methods to induce mitophagy and a variety of assays to monitor this process. With these new methods, the research on mitophagy has been developed rapidly. In particular, some key receptors and regulatory factors in fungi have been identified, which provides a basis for further understanding of the mechanism of this process. Although it has been studied extensively in the model yeast Saccharomyces cerevisiae, mitophagy in pathogenic fungi remains poorly understood. However recent studies have shown that mitophagy is involved in the regulation of pathogenicity of pathogenic fungi, which greatly increases the importance of mitophagy. Therefore, it is necessary to review the current research on mitophagy in order to provide an accurate understanding of mitophagy and promote mitophagy research in the pathogenic fungi.
\end{abstract}

\section{Keywords}

Mitochondria, Mitophagy, Yeast, Pathogenic Fungi

\section{Introduction}

Mitochondria, double-membrane-enclosed organelles, carry out most of the cellular oxidative processes and produce the bulk of the cell's adenosine triphosphate (ATP), which is the primary source of energy [1] [2]. Moreover, mitochondria are important organelles in eukaryotic cells, which are involved in vital functions that include cellular differentiation, cell signaling, and cell death, as well as maintaining control of the cell cycle and cell growth [3]. Mitochondri- 
al dysfunction is usually multifactorial and characterized by abnormal accumulation of ATP and reactive oxygen species (ROS) [1] [3]. Thereby dysfunctional mitochondria may disrupt the regulation of intracellular cell homeostasis, and be harmful to the cell [4]. Mitochondria possess some internal quality control machinery, but an important contribution for quality control and clearance of mitochondria comes from mitophagy, which is a selective degradation of mitochondria by autophagy [5]. The primary research goal of mitophagy is to elucidate the molecular mechanisms by which cells maintain a certain population of healthy and functional mitochondria for normal cellular metabolism [5] [6].

In the past two decades, many new methods have been used for the study of mitophagy, and great progress has been made in studying the roles of mitophagy in fungi, especially in a model yeast $S$. cerevisiae [6]-[14]. Recently, a particular interest has been devoted to study mitophagy in the pathogenic fungi [15]-[20]. This review summarizes the research progress in mitophagy, including methods to induce mitophagy, a variety of assays to monitor mitophagy, and the molecular mechanism of mitophagy, with an emphasis on studies performed in the pathogenic fungal species Candida glabrata, Candida albicans, Magnaporthe oryzae, Aspergillus oryzae, Ustilago maydis and Beauveria bassiana. This review will provide a better understanding of the role of mitophagy in fungal pathogenicity and help develop new strategies for fungal disease control.

\section{Induction and Physiological Roles of Mitophagy in Yeast and Pathogenic Fungi}

\subsection{Induction of Mitophagy}

Eukaryotes perceive nutritional status and activate autophagy to survive during starvation [13]. Several conditions, including nitrogen starvation, stationary phase, rapamycin treatment, and some alternation of mitochondrial function, induce mitophagy in fungi [5] [19] [21] [22] [23] [24] [25]. In yeast and pathogenic fungi, nitrogen starvation is the most common condition for induction of mitophagy [12] [23] [24] [26]. In yeasts, mitophagy is generally observed during the post-logarithmic growth phase when cells are cultured in a respiratory medium containing a non-fermentable carbon source, such as lactate and glycerol, or pre-cultured in a non-fermentable medium and then transferred to a nitrogen-free fermentation medium containing glucose [5] [6] [21] [25] [27] [28]. Moreover, there are variations in the conditions of mitophagy induction among different species of yeast. In the budding yeast $S$. cerevisiae, mitophagy is immediately induced when cells are cultured under nitrogen-starved conditions after pre-culturing in a non-fermentable medium [8] [21] [29] [30] [31]. Interestingly, in $S$. cerevisiae, mitophagy is also induced by rapamycin treatment. Rapamycin could inhibit the TOR (target of rapamycin) signaling pathway to regulate respiration induced mitophagy [28]. In the haploid budding yeast $C$. glabrata, a close relative of $S$. cerevisiae, mitophagy can be induced in long-term culture in medium containing respiratory carbon source, but not under short-period ni- 
trogen starvation condition [22]. The mechanism by which mitophagy is suppressed in media containing a respiratory carbon source remains to be clarified. As for, C. albicans, a methylotrophic yeast, mitophagy is also induced under nitrogen starvation conditions [26]. These studies have indicated physiological differences underlying mitophagy regulation in different yeast species.

Induction of mitophagy, in the filamentous fungus $M$. oryzae (rice blast fungus), is observed under nitrogen starvation [17] [23]. In addition, mitochondria are one of the major sources of ROS in cells, imbalance of ROS levels and the resulting oxidative stress within these organelles is an attractive candidate for an inducer of mitophagy [4]. In M. oryzae, oxidative stress is another inducer of mitophagy in addition to minimal medium with glycerol [23]. Likewise, in another filamentous fungus Aspergillus oryzae, mitophagy can be induced in the minimal medium with lactic acid or glycerol as the sole carbon source [32]. Mitophagy in a highly attractive model fungus $U$. maydis for the study of cellular processes is detected after the transfer from log-phase cells to either water-diluted YEPS (yeast extract-peptone-sucrose) or nitrogen-deprived minimal medium (MMG-N; glucose is the sole carbon source), which are amino acid starvation conditions [19]. Additionally, mitophagy is also induced as response to conditional overexpression of the a2 mating-type locus gene, which induces mitochondrial dysfunction in $U$. maydis [33]. Recently, a study showed that the mitophagy of $B$. bassiana, a filamentous entomopathogenic fungus, was induced by mild starvation [31]. Variations in the different fungi growth environments and fungal diversity can explain the differences in the mitophagy-inducing conditions for these fungi. However, the mechanism of these different conditions to trigger mitophagy needs further study.

In addition, there are different types of mitophagy occurring during nutrient deprivation, mitochondrial damage and at last the form of self-eating mitochondria. Nutrient deprivation and mitochondrial damage mitophagy are known as the macro-mitophagy while the latter is known as the micro-mitophagy. Micro-mitophagy is well characterized in yeast. Different molecular mechanisms are required for different types of mitophagy. However to elucidate the molecular mechanism of the various forms of mitophagy in yeast and pathogenic fungi, more detailed research needs to be conducted [34].

\subsection{The Physiological Role of Mitophagy}

The physiological roles of mitophagy have been recently determined using cells with mitophagy deficiency, such as ATG32, ATG24 and ATG26 deletion mutants in different fungi [23] [29] [32]. In S. cerevisiae and C. glabrata, mitophagy plays an essential role in maintaining the appropriate quantity and quality of mitochondria to minimize the ROS production when cells encounter starvation stress in respiratory growth [12] [22]. Mitochondrial membrane potential (MMP) is critical for maintaining the physiological function of respiratory chain to generate ATP and has an important impact on mitochondrial function and 
cellular longevity [31]. It has also been reported that mitophagy is required for maintenance of MPP to sustain the levels of intracellular ATP and retaining the longevity in S. cerevisiae and C. glabrata [6] [22]. In addition, decreased or dysregulated mitophagy can also contribute to the decline in mitochondrial quality and function, which is related to aging [6]. However, how mitophagy is regulated during aging in most of the fungi remains to be elucidated.

In filamentous fungi, mitophagy plays essential roles during differentiation in sexual and asexual development, and pathogenesis [23] [32]. For example, mitophagy is essential for conidia differentiation and fungal pathogenesis in $M$. oryzae [17] [23]. Similarly, in A. oryzae, strongly reduced conidial and aerial hyphae growth and invasive growth were shown in the ATG26 deletion strain, which is mitophagy deficient mutant [32]. Moreover, in B. bassiana, mitophagy is related to the development of blastopores and starvation stress [20]. However, it has been suggested that mitophagy defects do not lead to serious defects in fungal pathogenicity in some pathogenic fungi, in contrast to the situation with non-specific autophagy [11] [17] [20] [23] [32]. The relationship between mitophagy and fungal pathogenesis remains elusive and needs to be further explored.

\section{Methods to Monitor Mitophagy in Yeast and Pathogenic Fungi}

\subsection{Transmission Electron Microscopy Analysis of Mitophagy}

Since the first evidence of mitophagy in yeast was reported by Kissova et al. [34], a considerable amount of work has been conducted in mitophagy. Methods for detecting mitophagy in fungi include transmission electron microscopy (TEM), fluorescence microscopy for co-localization of mitochondria with autophagosomes, and western blotting to quantify degradation of mitochondrial proteins [35] [36] [37]. TEM is a widely used methodology to detect mitophagy [36]. TEM observations make it possible to distinguish between selective mitophagy and nonselective mitochondrial degradation and qualitatively distinguish early stages of mitophagy by the presence of autophagosomes containing intact mitochondria from late stages of mitophagy [38]. Although TEM requires expertise in recognizing and analyzing these ultrastructural features, this approach has higher resolution than fluorescence-based imaging technology [36] [37]. Nonetheless, TEM is best used in combination with other methods to ensure the complex and holistic approach that is becoming increasingly necessary in mitophagy research.

\subsection{Fluorescence Microscopy for Analyzing Mitophagy}

Fluorescence microscopy is also an important and commonly used methodology for assessing and quantifying the complete degradation of mitochondria by mitophagy [23] [32]. Mitophagy can be visualized under fluorescence microscopy using GFP/RFP/mCherry-tagged mitochondrial proteins or dye-labeled mitochondria [17] [23] [32] [39]. This type of microscopy has been commonly used 
in most of the studies detecting mitophagy in yeast and pathogenic fungi [17] [23] [32] [39] [40] [41]. In this assay, when mitophagy is induced, the damaged mitochondria are transported to tagged or labeled vacuoles, and the mitophagy can be observed by the fluorescence signals from GFP/RFP/mCherry or dyes in the vacuoles [17] [23] [27] [32] [34] [35] [39]. Observing mitophagy using fluorescence microscope could provide results for mitophagy observation in a large number of cells.

\subsection{Western Blot Analysis of Mitophagy}

Mitophagy can also be quantified by the degradation of mitochondrial proteins using Western blot assay. This assay is more appropriate and specific for mitophagy [42]. However, high levels of mitophagy may be required to detect mitochondrial protein degradation, and changes in protein levels can be seriously affected by other factors, for example, by proteasomal degradation pathways and alterations in biogenesis [37]. The outer mitochondrial membrane proteins are degraded by both autophagy and the proteasome, which makes interpretation of data from Western blot assays very complicated [43]. In addition, this method of quantification may underestimate mitophagy when substantial mitochondrial biosynthesis is occurring simultaneously [11] [43]. According to published research results, mitochondrial inner membrane or matrix proteins have been recommended for mitophagy assessment by Western blot analysis in fungi as it has been more objective and quantitative [35].

In conclusion, at present, no single experimental method is sufficient for the assessment of mitophagy, because none of these assays alone provide the evidence to prove the occurrence of cargo-specific mitophagy. Consequently, it is important to use a variety of assays to study mitophagy.

\section{The Molecular Mechanism of Mitophagy in Yeast and Pathogenic Fungi}

\subsection{Receptor Proteins Involved in Mitophagy in Different Fungi}

Selective autophagy pathway requires binding of the cargo receptor to the core autophagy machinery that linking the cargo to the phagophore [5] [30] [44]. In mitophagy, the receptor recruits the autophagy machinery components directly to the superfluous or damaged mitochondria to the autophagosome membrane to initiate selective autophagy [8] [9] [29] [30]. The receptor-proteins functions related to mitophagy in yeasts and pathogenic fungi were summarized in Table 1. Among them, $S$. cerevisiae Atg32 is the first mitophagy receptor identified [29]. Moreover, the Atg32 homolog also was identified as a mitophagy receptor in the pathogenic yeast C. glabrata [45]. In addition, several mitophagy receptors were identified, including MoAtg24 in M. oryzae and AoAtg26 in A. oryzae, which are directly involved in mitophagy similar to the yeast Atg32 [23] [32]. Notably, AoAtg26 is involved in both mitophagy and pexophagy in A. oryzae [32]. At present, how they perceive the mitochondrial stresses within mitochondria 
Table 1. Summary of the mitophagy receptors and key proteins in yeasts and pathogenic fungi.

\begin{tabular}{|c|c|c|c|c|c|c|}
\hline Protein & Species & Host & Localized & Function & Induction condition & Ref. \\
\hline \multirow[t]{2}{*}{$\begin{array}{l}\text { ATG32 } \\
\text { (Mitochondria-anchor } \\
\text { receptor) }\end{array}$} & S. cerevisiae & N.A & $\begin{array}{l}\text { Outer } \\
\text { membrane of } \\
\text { Mitochondria }\end{array}$ & $\begin{array}{l}\text { Recruits the autophagic } \\
\text { machinery components to } \\
\text { mitochondria and regulates } \\
\text { degradation of mitochondria }\end{array}$ & $\begin{array}{l}\text { Nitrogen starvation } \\
\text { conditions and } \\
\text { non-fermentable carbon } \\
\text { source }\end{array}$ & [25] [29] [30] [45] \\
\hline & C. glabrata & Human & Mitochondria & $\begin{array}{l}\text { Contribute to mitochondrial } \\
\text { quality control mechanism, } \\
\text { longevity of cells and } \\
\text { pathogenesis }\end{array}$ & Iron-depleted conditions & {$[22][38][46]$} \\
\hline $\begin{array}{l}\text { ATG24 (Sorting } \\
\text { nexin) }\end{array}$ & M. oryzae & $\begin{array}{l}\text { Rice and } \\
\text { wheat }\end{array}$ & Mitochondria & $\begin{array}{l}\text { Required for sporulation and } \\
\text { proper invasive growth during } \\
\text { rice blast infection process }\end{array}$ & $\begin{array}{l}\text { Starvation and oxidative } \\
\text { stress }\end{array}$ & [17] [23] [47] \\
\hline $\begin{array}{l}\text { ATG26 (Sterol } \\
\text { glucosyltransferase) }\end{array}$ & A. oryzae & $\begin{array}{l}\text { Rice, maize, } \\
\text { barley and } \\
\text { wheat }\end{array}$ & PAS & $\begin{array}{l}\text { Required for selective autophagy } \\
\text { degradation of mitochondria, } \\
\text { peroxisome and nuclei } \\
\text { differentiation process }\end{array}$ & $\begin{array}{l}\text { Non-fermentable carbon } \\
\text { source }\end{array}$ & {$[32]$} \\
\hline $\begin{array}{l}\text { ATG8 (Ubiquitin like } \\
\text { protein) }\end{array}$ & $\begin{array}{l}\text { S. cerevisiae, } \\
\text { C. glabrata, } \\
\text { M. oryzae, } \\
\text { A. oryzae }\end{array}$ & $\begin{array}{l}\text { Human, } \\
\text { Rice, wheat, } \\
\text { barley and } \\
\text { maize }\end{array}$ & $\begin{array}{l}\text { Autophagosom } \\
\text { al membrane }\end{array}$ & $\begin{array}{l}\text { mediating the recruitment of } \\
\text { different cargo molecules into } \\
\text { autophagosomes }\end{array}$ & $\begin{array}{l}\text { Starvation, oxidative } \\
\text { stress Non-fermentable } \\
\text { carbon source }\end{array}$ & $\begin{array}{l}{[25][29][30][45]} \\
{[22][38][46][17]} \\
{[23][47][32]}\end{array}$ \\
\hline $\begin{array}{l}\text { ATG11 } \\
\text { (Scaffold protein) }\end{array}$ & $\begin{array}{l}\text { S. cerevisiae, } \\
\text { C. glabrata, } \\
\text { M. oryzae, } \\
\text { A. oryzae }\end{array}$ & $\begin{array}{l}\text { Human, } \\
\text { Rice, wheat, } \\
\text { barley and } \\
\text { maize }\end{array}$ & Cytosol & $\begin{array}{l}\text { an adaptor protein for selective } \\
\text { autophagy }\end{array}$ & $\begin{array}{l}\text { phosphate } \\
\text { starvation-induced } \\
\text { autophagy }\end{array}$ & $\begin{array}{l}{[25][29][30][45]} \\
{[22][38][46][17]} \\
{[23][47][32]}\end{array}$ \\
\hline
\end{tabular}

to activate mitophagy remains largely unknown in pathogenic fungi.

\subsection{Key Proteins in the Mechanism Process of Mitophagy in Yeasts and Pathogenic Fungi}

Various fungi share similar molecular mechanism for mitophagy. The receptor protein interacts with scaffold protein Atg11 to organize the core autophagic machinery at the phagophore assembly site (PAS), and mediates recruitment of Atg8, which initiates phagophore elongation from the PAS [5] [48]. In S. cerevisiae, the interaction of Atg32 and Atg11 is regulated by phosphorylation of Atg32 [25]. As observed in S. cerevisiae, the phosphorylation of CgAtg32 before initiation of mitophagy was also detected in iron-depleted C. glabrata cells [22]. In addition, the Atg11 homologs have been identified in $M$. oryzae, A. oryzae and $U$. maydis, but the requirement of Atg11 for mitophagy has only been elucidated in S. cerevisiae, A. oryzae and U. maydis [18] [19] [26] [39] [49] [50] [51]. The role of Atg11 in the initiation of mitophagy in other fungi remains to be studied.

In mitophagy, the receptor recruits the core autophagy machinery components, which are required for nonselective and other selective autophagy, directly to the superfluous or damaged mitochondria to initiate selective autophagy [5]. An ubiquitin-like protein Atg8 is one of the core elements in autophagy. Atg8 interacts with mitophagy receptors through a conserved WXXL-like se- 
quence (W/Y-X-X-L/I/V), Atg8-family-interacting motif (AIM), to mediate selective recognition of the superfluous or damaged mitochondria by the phagophore [30]. Subsequently, an autophagosome, which completely encloses the mitochondria, forms to depolarize and degrade mitochondria [10]. Studies in $S$. cerevisiae showed that the interaction between Atg32 and Atg8 facilitates the formation of autophagosomes surrounding the mitochondria and anchor the mitochondria to autophagosomes during mitophagy process. The function of Atg8 in autophagy has been investigated in yeast and several pathogenic fungi including S. cerevisiae, M. oryzae, A. oryzae, U. maydis and B. bassiana [52] [53] [54] [55], indicating that the Atg8 protein has different roles in pathogenic fungi. However, the role of Atg8 in mitophagy in pathogenic fungi remains elusive. The formation of the autophagosome, surrounding a mitochondria proceeds by the means of Atg1 complex activating the phosphatidylinositol 3-kinase (PI3K) complex involving the Vps15, Vps34, Atg6, and Atg14 proteins [5].

Moreover, mutants of the corresponding core autophagy genes, which are involved in mitophagy, showed defects in the conidiation, growth, appressorium formation or virulence in both yeast and pathogenic fungi. For example in $C$. glabrata, ATG17 mutant showed reduced survival in host tissues [46]. Except MoATG13 and MoATG17, the deletion of the core ATG genes (MoATG1MoATG12, MoATG14-16, MoATG18) resulted in the loss of pathogenicity in $M$. oryzae [56]. In addition, disruption of core autophagy genes (AoATG4, AoATG8, AoATG13 and AoATG15) in A. oryzae resulted in serious defects in the development of aerial hyphae and conidia [57]. Figure 1 has given a schematic representation of the involvement of the receptor proteins and key proteins in mitophagy process in yeast and pathogenic fungi.

It is also worth noting that the mitochondrial fusion and fission process, especially the mitochondrial fission process, affects mitophagy. It is known that mitochondria fission machinery is active after prolonged nutrient stress, which leads to degradation through mitophagy [17]. In S. cerevisiae, mitochondrial fission is executed by the dynamin-related GTPase Dnm1 which binds to the outer membrane via Fis1 and Mdv1 [59]. Notably, the Dnm1-Atg11 interaction is required for inducing of mitochondrial fission and mitophagy in $S$. cerevisiae. However, it is not clear whether fission is required for mitophagy in $S$. cerevisiae due to the contradictory results from different studies [60]. In M. oryzae, disruption of mitochondria fission machinery gene, such as DNM1, MDV1 and FIS1, affects both mitophagy and nonspecific autophagy and causes significant reduction in pathogenicity [17]. In addition, Dnm1 fission component is required for lga2-induced mitophagy, but not for starvation-induced mitophagy in $U$. maydis, suggesting that Dnm1-dependent fission is not always required for mitophagy [33]. A recent study showed that Fis1, Mdv1 and Dnm1 are indispensable for mitochondrial fusion and contribute to mitophagy in B. bassiana [20]. As opposed to fission, mitochondria fusion machinery consists of three key proteins, Fzo1, Ugo1 and Mgm1, in S. cerevisiae [61]. Deletion of S. cerevisiae FZO1 


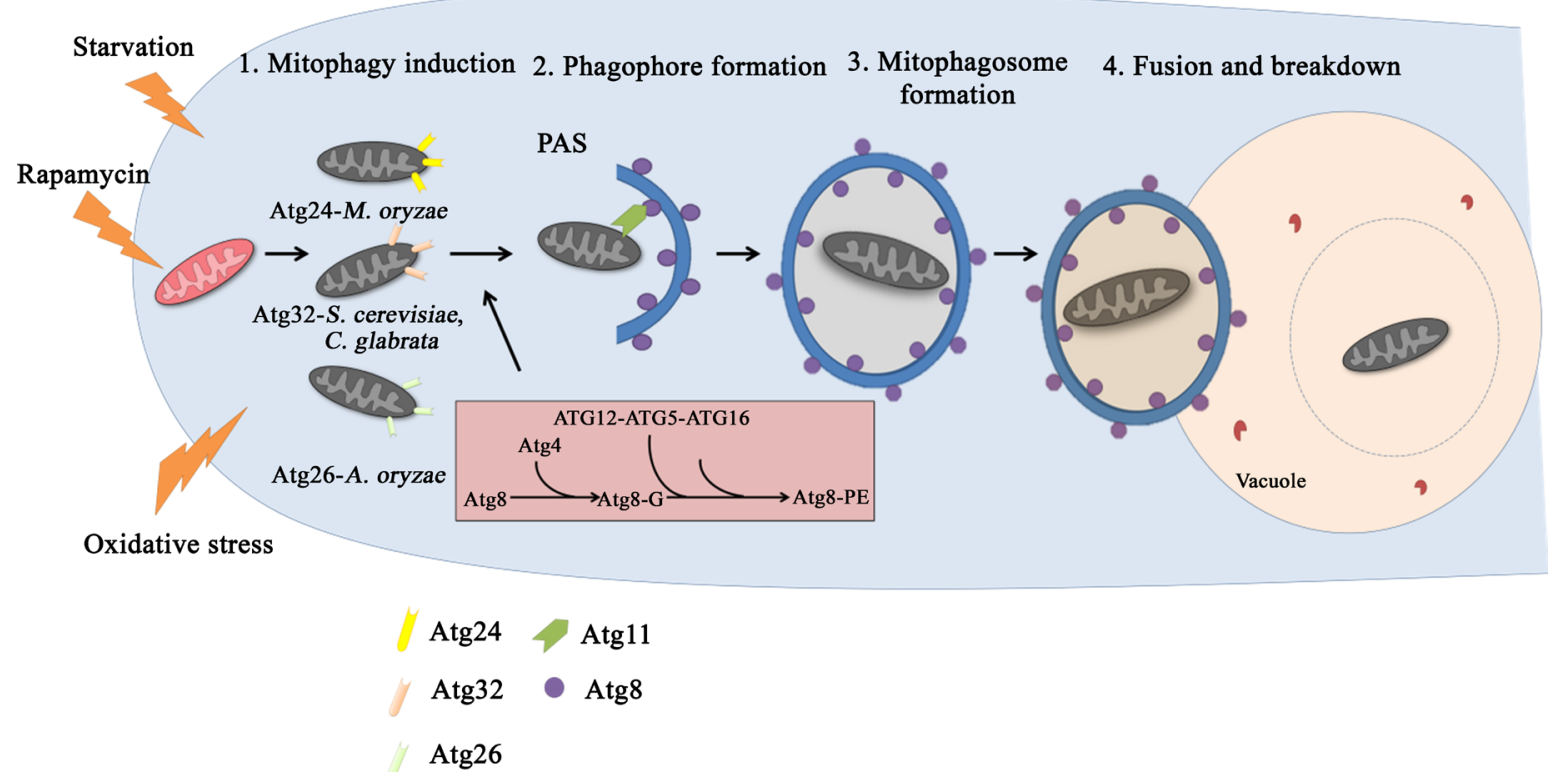

Figure 1. Schematic representation of the involvement of Atg32, Atg24 and Atg26 in mitophagy in yeast and pathogenic fungi. 1. Upon induction of mitophagy, damaged mitochondria are tagged for degradation by mitophagy receptors, Atg32 in S. cervisiae and C. glabrata, Atg24 in M. oryzae and Atg26 in A. oryzae [23] [29] [32]. Atg11 and Atg8 bind to the specific mitophagy receptor to recruit mitochondria to the PAS, where mitophagy-specific uptaking occurs [58]. 2. The formation of the autophagosome, proceeds by the means of the mitopahgy specific receptor and Atg11 Atg8 interaction. [13]. 3. Subsequently, a double (or multi-) membrane layer starts to sequester the tagged mitochondria, resulting in the formation of a mitophagosome. 4. Upon completion of sequestration, the outer membrane layer of the mitophagosome fuses with the vacuolar membrane. The inner membrane of the mitophagosome, along with the sequestered autophagic body, is degraded. At last the resulting metabolites are recycled to the cytoplasm for further use [59].

reduces mitophagy [61] [62], suggesting that mitochondrial fusion process also affects mitophagy. In conclusion, these results indicate that the fission-fusion machinery is related to mitophagy in yeast and pathogenic fungi, but the role of mitochondrial fusion and fission factors in mitophagy is still not consisted.

\section{Conclusion and Future Directions}

Mitophagy is of great importance in scavenging damaged mitochondria to maintain a stable cellular state [6]. The studies in the model organism S. cerevisiae and pathogenic fungi have promoted our understanding of the physiological role and molecular mechanism of mitophagy in eukaryotic cells [5] [6] [21] [29] [30]. Mitophagy has been implicated in various physiological and pathological conditions including maintaining the appropriate quantity and quality of mitochondria related to aging and development. Recent studies have shown that mitophagy is involved in the regulation of pathogenicity of pathogenic fungi, which greatly increases the importance of mitophagy. Furthermore, the latest findings about mitophagy have highlighted special receptor and key proteins required for mitophagy in fungi. For further understanding of mitophagy in pathogenic fungi, several questions remain to be answered. For example, how does mitosis integrate stress and developmental signals to meet precise cellular needs? How 
does receptor mediated mitophagy selectively remove unwanted or damaged mitochondria in order to regulate the quality and quantity of mitochondria? Is the mitophagy signaling pathway consistent among yeast and pathogenic fungi? How are mitophagy and fusion-fission mechanisms connected? Whether there is more accurate monitoring of the whole process of mitosis is helpful to improve the understanding of mitophagy and explore its physiological and pathological effects. The answers to these questions will significantly enhance our knowledge of mitophagy and provide much needed insights into the pathogenesis of pathogenic fungi, thus helping to identify potential fungal disease control strategies. In pathogenic fungi, future research in mitophagy remains interesting and we look forward to more significant insights.

\section{Acknowledgements}

This project was supported by the National Natural Science Foundation of China (32000103 and 31900127), the Chinese Academy of Agricultural Sciences under the "Elite Youth" program, the Agricultural Sciences and Technologies Innovation Program, Zhejiang Provincial Natural Science Foundation of China (LQ19C130007), key R\&D project of Zhejiang Province (2019C02018), and Central Public-interest Scientific Institution Basal Research Fund of China National Rice Research Institute (grant No. 2017RG002-8).

\section{Conflicts of Interest}

The authors declare no conflicts of interest regarding the publication of this paper.

\section{References}

[1] Chinopoulos, C. and Adam-Vizi, V. (2010) Mitochondria as ATP Consumers in Cellular Pathology. Biochimica et Biophysica Acta, 1802, 221-227. https://doi.org/10.1016/j.bbadis.2009.08.008

[2] McBride, H.M., Neuspiel, M. and Wasiak S. (2006) Mitochondria: More than Just a Powerhouse. Current Biology, 16, 551-560. https://doi.org/10.1016/j.cub.2006.06.054

[3] Osellame, L.D., Blacker, T.S. and Duchen, M.R. (2012) Cellular and Molecular Mechanisms of Mitochondrial Function. Best Practice \& Research Clinical Endocrinology \& Metabolism, 26, 711-723. https://doi.org/10.1016/j.beem.2012.05.003

[4] Zorov, D.B., Juhaszova, M. and Sollott, S.J. (2014) Mitochondrial Reactive Oxygen Species (ROS) and ROS-Induced ROS Release. Physiological Reviews, 94, 909-950. https://doi.org/10.1152/physrev.00026.2013

[5] Youle, R.J. and Narendra, D.P (2011) Mechanisms of Mitophagy. Nature Reviews Molecular Cell Biology, 12, 9-14. https://doi.org/10.1038/nrm3028

[6] Fukuda, T. and Kanki, T. (2018) Mechanisms and Physiological Roles of Mitophagy in Yeast. Molecules and Cells, 41, 35-44. https://doi.org/10.14348/molcells.2018.2214

[7] Ding, W.X. and Yin, X.M. (2012) Mitophagy: Mechanisms, Pathophysiological Roles, and Analysis. Biological Chemistry, 393, 547-564. 
https://doi.org/10.1515/hsz-2012-0119

[8] Kanki, T., Furukawa, K. and Yamashita, S. (2015) Mitophagy in Yeast: Molecular Mechanisms and Physiological Role. Biochimica et Biophysica Acta (BBA)-Molecular Cell Research, 1853, 2756-2765. https://doi.org/10.1016/j.bbamcr.2015.01.005

[9] Novak, I. (2012) Mitophagy: A Complex Mechanism of Mitochondrial Removal. Antioxidants \& Redox Signaling, 17, 794-802. https://doi.org/10.1089/ars.2011.4407

[10] Feng, Y.C., He, D., Yao, Z.Y. and Klionsky, D.J. (2014) The Machinery of Macroautophagy. Cell Research, 24, 24-41. https://doi.org/10.1038/cr.2013.168

[11] Klionsky, D.J. (2005) The Molecular Machinery of Autophagy: Unanswered Questions. Journal of Cell Science, 118, 7-18. https://doi.org/10.1242/jcs.01620

[12] Kurihara, Y., Kanki, T., Aoki, Y., Hirota, Y., Saigusa, T. and Uchiumi, T. (2012) Mitophagy Plays an Essential Role in Reducing Mitochondrial Production of Reactive Oxygen Species and Mutation of Mitochondrial DNA by Maintaining Mitochondrial Quantity and Quality in Yeast. Journal of Biological Chemistry, 287, 3265-3272. https://doi.org/10.1074/jbc.M111.280156

[13] Parzych, K.R. and Klionsky, D.J. (2014) An Overview of Autophagy: Morphology, Mechanism, and Regulation. Antioxidants \& Redox Signaling, 20, 460-473. http://doi.org/10.1089/ars.2013.5371

[14] Yang, Z. and Klionsky, D.J. (2009) An Overview of the Molecular Mechanism of Autophagy. In: Levine, B., Yoshimori, T. and Deretic, V., Eds., Autophagy in Infection and Immunity, Current Topics in Microbiology and Immunology, Vol. 335, Springer, Berlin, Heidelberg, 1-32. https://doi.org/10.1007/978-3-642-00302-8 1

[15] Kershaw, M.J. and Talbot, N.J. (2009) Genome-Wide Functional Analysis Reveals That Infection-Associated Fungal Autophagy Is Necessary for Rice Blast Disease. Proceedings of the National Academy of Sciences of the United States of America, 106, 15967-15972. https://doi.org/10.1073/pnas.0901477106

[16] Khan, I.A., Lu, J.-P., Liu, X.-H., Rehman, A. and Lin, F.-C. (2012) Multifunction of Autophagy-Related Genes in Filamentous Fungi. Microbiological Research, 167, 339-345. https://doi.org/10.1016/j.micres.2012.01.004

[17] Kou, Y.J., He, Y.L., Qui, J.H., Shu, Y.Z., Yang, F.F., Deng, Y.Z. and Naqvi, N.I. (2019) Mitochondrial Dynamics and Mitophagy Are Necessary for Proper Invasive Growth in Rice Blast. Molecular Plant Pathology, 20, 1147-1162. https://doi.org/10.1111/mpp.12822

[18] Tadokoro, T., Kikuma, T. and Kitamoto, K. (2015) Functional Analysis of AoAtg11 in Selective Autophagy in the Filamentous Fungus Aspergillus oryzae. Fungal Biology, 119, 560-567. https://doi.org/10.1016/j.funbio.2015.03.001

[19] Wagner-Vogel, G., Lämmer, F., Kämper, J. and Basse, C.W. (2015) Uniparental Mitochondrial DNA Inheritance Is Not Affected in Ustilago Maydis Deltaatg11 Mutants Blocked in Mitophagy. BMC Microbiology, 15, Article No. 23. https://doi.org/10.1186/s12866-015-0358-Z

[20] Wang, J.J., Peng, Y.J., Ding, J.L., Feng, M.G. and Ying, S.H. (2020) Mitochondrial Fission Is Necessary for Mitophagy, Development and Virulence of the Insect Pathogenic Fungus Beauveria bassiana. Journal of Applied Microbiology, 129, 411-421. https://doi.org/10.1111/jam.14619

[21] Muller, M., Lu, K. and Reichert, A.S. (2015) Mitophagy and Mitochondrial Dynamics in Saccharomyces cerevisiae. Biochimica et Biophysica Acta (BBA)-Molecular Cell Research, 1853, 2766-2774. https://doi.org/10.1016/j.bbamcr.2015.02.024 
[22] Nagi, M., Tanabe, K., Nakayama, H., Ueno, K., Yamagoe, S., Umeyama, T., Ohno, H. and Miyazaki, Y. (2016) Iron-Depletion Promotes Mitophagy to Maintain Mitochondrial Integrity in Pathogenic Yeast Candida glabrata. Autophagy, 12, 1259-1271. https://doi.org/10.1080/15548627.2016.1183080

[23] He, Y.L., Deng, Y.Z. and Naqvi, N.I. (2013) Atg24-Assisted Mitophagy in the Foot Cells Is Necessary for Proper Asexual Differentiation in Magnaporthe oryzae. Autophagy, 9, 1818-1827. https://doi.org/10.4161/auto.26057

[24] Henkel, V., Schürmanns, L., Brunner, M., Hamann, A. and Osiewacz, H.D. (2020) Role of Sorting Nexin PaATG24 in Autophagy, Aging and Development of Podospora anserina. Mechanisms of Ageing and Development, 186, Article ID: 111211. https://doi.org/10.1016/j.mad.2020.111211

[25] Levchenko, M., Lorenzi, I. and Dudek, J. (2016) The Degradation Pathway of the Mitophagy Receptor Atg32 Is Re-Routed by a Posttranslational Modification. PLoS ONE, 11, e0168518. https://doi.org/10.1371/journal.pone.0168518

[26] Cui, L.F., Zhao, H., Yin, Y.J., Liang, C., Mao, X.L., Liu, Y.Z., Yu, Q.L. and Li, M.C. (2019) Function of Atg11 in Non-Selective Autophagy and Selective Autophagy of Candida albicans. Biochemical and Biophysical Research Communications, 516, 1152-1158. https://doi.org/10.1016/j.bbrc.2019.06.148

[27] Bhatia-Kissova, I. and Camougrand, N. (2010) Mitophagy in Yeast: Actors and Physiological Roles. FEMS Yeast Research, 10, 1023-1034. https://doi.org/10.1111/j.1567-1364.2010.00659.x

[28] Furukawa, K., Innokentev, A. and Kanki, T. (2019) Regulatory Mechanisms of Mitochondrial Autophagy: Lessons from Yeast. Frontiers in Plant Science, 10, 1479. https://doi.org/10.3389/fpls.2019.01479

[29] Kanki, T., Wang, K., Cao, Y., Baba, M. and Klionsky, D.J. (2009) Atg32 Is a Mitochondrial Protein That Confers Selectivity during Mitophagy. Developmental Cell, 17, 98-109. https://doi.org/10.1016/j.devcel.2009.06.014

[30] Okamoto, K., Kondo-Okamoto, N. and Ohsumi, Y. (2009) Mitochondria-Anchored Receptor Atg32 Mediates Degradation of Mitochondria via Selective Autophagy. Developmental Cell, 17, 87-97. https://doi.org/10.1016/j.devcel.2009.06.013

[31] Kikuma, T., Tadokoro, T., Maruyama, J. and Kitamoto, K. (2017) AoAtg26, a Putative Sterol Glucosyltransferase, Is Required for Autophagic Degradation of Peroxisomes, Mitochondria, and Nuclei in the Filamentous Fungus Aspergillus oryzae. Bioscience, Biotechnology, and Biochemistry, 81, 384-395. https://doi.org/10.1080/09168451.2016.1240603

[32] Nieto-Jacobo, F., Pasch, D. and Basse C.W. (2012) The Mitochondrial Dnm1-Like Fission Component Is Required for Iga2-Induced Mitophagy but Dispensable for Starvation-Induced Mitophagy in Ustilago maydis. Eukaryotic Cell, 11, 1152-1166. https://doi.org/10.1128/EC.00115-12

[33] Kissova, I., Deffieu, M., Manon, S. and Camougrand, N. (2004) Uth1p is Involved in the Autophagic Degradation of Mitochondria. Journal of Biological Chemistry, 279, 39068-39074. https://doi.org/10.1074/jbc.M406960200

[34] Camougrand, N., Kissova, I., Salin, B. and Devenish, R.J. (2008) Chapter 8 Monitoring Mitophagy in Yeast. In: Klionsky, D.J., Ed., Autophagy: Lower Eukaryotes and Non-Mammalian Systems, Part A, Academic Press, Cambridge, Massachusetts, 89-107. https://doi.org/10.1016/S0076-6879(08)03208-4

[35] Chakraborty, J., Caicci, F., Roy, M. and Ziviani, E. (2020) Investigating Mitochondrial Autophagy by Routine Transmission Electron Microscopy: Seeing Is Believing? Pharmacological Research, 160, Article ID: 105097. 
https://doi.org/10.1016/j.phrs.2020.105097

[36] Dolman, N.J., Chambers, K.M., Mandavilli B., Batchelor, R.H. and Janes, M.S. (2013) Tools and Techniques to Measure Mitophagy Using Fluorescence Microscopy. Autophagy, 9, 1653-1662. https://doi.org/10.4161/auto.24001

[37] Tanabe, K. and Nagi, M. (2018) Monitoring of Iron Depletion-Induced Mitophagy in Pathogenic Yeast. In: Hattori, N. and Saiki, S., Eds., Mitophagy, Methods in Molecular Biology, Vol. 1759, Humana Press, New York, 161-172. https://doi.org/10.1007/7651 2017 40

[38] Farre, J.C., Burkenroad, A., Burnett, S.F. and Subramani, S. (2013) Phosphorylation of Mitophagy and Pexophagy Receptors Coordinates Their Interaction with Atg8 and Atg11. EMBO Reports, 14, 441-449. https://doi.org/10.1038/embor.2013.40

[39] Mijaljica, D., Prescott, M. and Devenish, R.J. (2011) A Fluorescence Microscopy Assay for Monitoring Mitophagy in the Yeast Saccharomyces cerevisiae. Journal of Visualized Experiments, No. 53, e2779. https://dx.doi.org/10.3791/2779

[40] Nagumo, S. and Okamoto, K. (2018) Investigation of Yeast Mitophagy with Fluorescence Microscopy and Western Blotting. In: Hattori, N. and Saiki, S., Eds., Mitophagy, Methods in Molecular Biology, Vol. 1759, Humana Press, New York, 71-83. https://doi.org/10.1007/7651_2017_11

[41] Eiyama, A. and K. Okamoto (2017) Assays for Mitophagy in Yeast. In: Mokranjac, D. and Perocchi, F., Eds., Mitochondria, Methods in Molecular Biology, Vol. 1567. Humana Press, New York, 337-347. https://doi.org/10.1007/978-1-4939-6824-4 20

[42] Malina, C., Larsson, C. and Nielsen, J. (2018) Yeast Mitochondria: An Overview of Mitochondrial Biology and the Potential of Mitochondrial Systems Biology. FEMS Yeast Research, 18, Article ID: foy040. https://doi.org/10.1093/femsyr/foy040

[43] Gatica, D., Lahiri, V. and Klionsky, D.J. (2018) Cargo Recognition and Degradation by Selective Autophagy. Nature Cell Biology, 20, 233-242. https://doi.org/10.1038/s41556-018-0037-Z

[44] Aihara, M. and Schwarz, T.L. (2014) Tor and the Sin3-Rpd3 Complex Regulate Expression of the Mitophagy Receptor Protein Atg32 in Yeast. Journal of Cell Science, 127, 3184-3196. https://doi.org/10.1242/jcs.153254

[45] Roetzer, A., Gratz, N., Kovarik, P. and Schuller, C. (2010) Autophagy Supports Candida glabrata Survival during Phagocytosis. Cell Microbiology, 12, 199-216. https://doi.org/10.1111/j.1462-5822.2009.01391.x

[46] Liu, X.H. and Lin, F.C. (2008) Investigation of the Biological Roles of Autophagy in Appressorium Morphogenesis in Magnaporthe oryzae. Journal of Zhejiang University-Science B, 9, 793-796. https://doi.org/10.1631/jzus.B0860013

[47] Xie, Z.P., Nair, U. and Klionsky, D.J. (2008) Atg8 Controls Phagophore Expansion during Autophagosome Formation. Molecular Biology of the Cell, 19, 3290-3298. https://doi.org/10.1091/mbc.e07-12-1292

[48] Matscheko, N., Mayrhofer, P., Rao, Y., Beier, V. and Wollert, T. (2019) Atg11 tethers Atg9 Vesicles to Initiate Selective Autophagy. PLoS Biology, 17, e3000377. https://doi.org/10.1371/journal.pbio.3000377

[49] Suzuki, H. and Noda, N.N. (2018) Biophysical Characterization of Atg11, a Scaffold Protein Essential for Selective Autophagy in Yeast. FEBS Open Biology, 8, 110-116. https://doi.org/10.1002/2211-5463.12355

[50] Zientara-Rytter, K. and Subramani, S. (2020) Mechanistic Insights into the Role of Atg11 in Selective Autophagy. Journal of Molecular Biology, 432, 104-122. https://doi.org/10.1016/j.jmb.2019.06.017 
[51] Kikuma, T., Takayuki, T., Maruyama, J. and Katsuhiko, K. (2006) Functional Analysis of the ATG8 Homologue Aoatg8 and Role of Autophagy in Differentiation and Germination in Aspergillus oryzae. Eukaryot Cell, 5, 1328-1336. https://doi.org/10.1128/EC.00024-06

[52] Liu, T.B., Liu, X.H., Lu, J.P, Zhang, L., Min, H. and Lin, F.C. (2010) The Cysteine Protease MoAtg4 Interacts with MoAtg8 and Is Required for Differentiation and Pathogenesis in Magnaporthe oryzae. Autophagy, 6, 74-85. https://doi.org/10.4161/auto.6.1.10438

[53] Liu, T.B., Liu, X.H., Lu, J.P., Zhang, L., Min, H. and Lin F.C. (2010) The Autophagy Genes atg8 and atg1 Affect Morphogenesis and Pathogenicity in Ustilago maydis. Molecular Plant Pathology, 11, 463-478. https://doi.org/10.1111/j.1364-3703.2010.00620.x

[54] Ying, S.H., Liu, J., Chu, X.L., Xie, X.Q. and Feng, M.G. (2016) The Autophagy-Related Genes BbATG1 and BbATG8 Have Different Functions in Differentiation, Stress Resistance and Virulence of Mycopathogen Beauveria bassiana. Science Reports, 6, Article No. 26376. https://doi.org/10.1038/srep26376

[55] Kikuma, T. and Kitamoto, K. (2011) Analysis of Autophagy in Aspergillus oryzae by Disruption of Aoatg13, Aoatg4, and Aoatg15 Genes. FEMS Microbiology Letter, 316, 61-69. https://doi.org/10.1111/j.1574-6968.2010.02192.x

[56] Kanki, T. and Klionsky, D.J. (2010) The Molecular Mechanism of Mitochondria Autophagy in Yeast. Molecular Microbiology, 75, 795-800. https://doi.org/10.1111/j.1365-2958.2009.07035.x

[57] Reggiori, F. and Klionsky, D.J. (2013) Autophagic Processes in Yeast: Mechanism, Machinery and Regulation. Genetics, 194, 341-361.

https://doi.org/10.1534/genetics.112.149013

[58] Scott, I. and Youle, R.J. (2010) Mitochondrial Fission and Fusion. Essays in Biochemistery, 47, 85-98. https://doi.org/10.1042/bse0470085

[59] Mendl, N., Occhipinti, A., Muller, M., Wild, P., Dikic, I. and Reichert, A.S. (2011) Mitophagy in Yeast Is Independent of Mitochondrial Fission and Requires the Stress Response Gene WHI. Journal of Cell Science, 124, 1339-1350. https://doi.org/10.1242/jcs.076406

[60] Merz, S., Hammermeister, M., Altman, K., Durr, M. and Westermann, B. (2007) Molecular Machinery of Mitochondrial Dynamics in Yeast. Biology Chemical, 388, 917-926. https://doi.org/10.1515/BC.2007.110

[61] Mao, K. and Klionsky, D.J. (2013) Mitochondrial Fission Facilitates Mitophagy in Saccharomyces cerevisiae. Autophagy, 9, 1900-1911.

https://doi.org/10.4161/auto.25804

[62] Jiang, J.C., Stumpferl, S.W. and Jazwinski, S.M. (2019) Dual Roles of Mitochondrial Fusion Gene FZO1 in Yeast Age Asymmetry and in Longevity Mediated by a Novel ATG32-Dependent Retrograde Response. Biogerontology, 20, 93-107. https://doi.org/10.1007/s10522-018-9779-Z 